
\title{
28 Research Square \\ An Exogenic Origin for the Volatiles Sampled by the LCROSS Impact
}

\section{Kathleen Mandt ( kathleen.mandt@jhuapl.edu )}

JHU/APL https://orcid.org/0000-0001-8397-3315

\section{Olivier Mousis}

Laboratoire d'Astrophysique de Marseille

\section{Dana Hurley}

Johns Hopkins University Applied Physics Laboratory

\section{Alexis Bouquet \\ Laboratoire d'Astrophysique de Marseille}

Kurt Retherford

Southwest Research Institute, San Antonio, TX, USA

\section{Lizeth Magana}

University of Texas at San Antonio \& Southwest Research Institute

\section{Adrienn Luspay-Kuti}

Johns Hopkins Applied Physics Laboratory https://orcid.org/0000-0002-7744-246X

\section{Article}

Keywords: volatiles, planetary science, LCROSS impact

Posted Date: September 8th, 2021

DOI: https://doi.org/10.21203/rs.3.rs-777152/v1

License: (c) (i) This work is licensed under a Creative Commons Attribution 4.0 International License. Read Full License

Version of Record: A version of this preprint was published at Nature Communications on February 8th, 2022. See the published version at https://doi.org/10.1038/s41467-022-28289-6. 


\section{Abstract}

Returning humans to the Moon presents an unprecedented opportunity to determine the origin of volatiles stored in the permanently shaded regions (PSRs), which trace the history of lunar volcanic activity, solar wind surface chemistry, and volatile delivery to the Earth and Moon through impacts of comets, asteroids, and micrometeoroids. So far, the source of the volatiles sampled by the Lunar Crater Observation and Sensing Satellite (LCROSS) plume $(1,2)$ has remained undetermined. We show here that the source could not be volcanic outgassing and the composition is best explained by cometary impacts. Ruling out a volcanic source means that volatiles in the top 13 meters of the Cabeus PSR regolith may be younger than the latest volcanic outgassing event ( $\sim$ billion years ago; Gya) (3).

\section{Introduction}

The LCROSS experiment impacted the upper stage of a spent Centaur rocket into the PSR of Cabeus crater, creating a plume that contained the first carbon-, nitrogen-, and sulfur-bearing volatiles detected in the lunar PSRs (1, 2, 4, 5). These ground-breaking observations not only provide ground truth for ongoing remote observations of water on the surface (e.g., 6, 7) and at depth (e.g., 8, 9), but provide vital clues to the origin of volatiles present on the Moon. The LCROSS plume was observed 30 seconds after impact by the Lunar Reconnaissance Orbiter (LRO) Lyman Alpha Mapping Project (LAMP), which detected $\mathrm{H}_{2}$ and $\mathrm{CO}(2,4)$. Meanwhile, the LCROSS shepherding spacecraft measured the abundance of several additional species relative to water for four minutes until it also impacted into Cabeus crater (1). The published abundances from $\operatorname{LAMP}(2,4)$ were derived from the expanding shell of vapor traveling at 3-4 km/s that passed LRO $>100 \mathrm{~km}$ away from the impact site. In contrast, the published abundances from the LCROSS shepherding spacecraft (1) were derived from vapor emanating from the impact site over time. Thus, the published LAMP observations were not made at the same time as the LCROSS measurements and require reanalysis for proper comparison (see supplemental materials).

To determine the origin of the volatiles observed in the LCROSS plume we must consider how volatile composition changed between the source and storage in the PSR. Several processes occur between initial delivery by the source and detection in the plume that change the molecular composition. This means that species that were measured in the plume may not be the same as the molecular species found in the source. To simplify the analysis and eliminate as many influences as possible, we instead compare the elemental composition of the LCROSS volatiles with the elemental composition of the potential sources, evaluating abundances of four elements as they relate to carbon: hydrogen $(\mathrm{C} / \mathrm{H})$, nitrogen $(\mathrm{N} / \mathrm{C})$, oxygen $(\mathrm{O} / \mathrm{C})$, and sulfur $(\mathrm{C} / \mathrm{S})$.

\section{Elemental Composition}

The elemental composition of the volatiles in the regolith of the PSR indicated by LCROSS observations depends on the type of ice storing the volatiles. We consider two cases based on types of ice that would be stable in the PSR regolith: condensates and clathrates. Condensates are volatiles condensed onto regolith grains, while clathrates are volatiles trapped in water cages. If the volatiles are stored as condensates, then each species is released according to its volatility temperature $(5,10)$, as assumed in $(4,11)$. The long-term stability of each species depends on how the temperature varies diurnally with depth (12). Thermal modeling shows that temperatures are stable below $\sim 0.2$ $m$ depth (13). The LCROSS impactor was estimated to have excavated material from 1-3 meters deep in the PSR (14), so the volatiles observed in the plume originated below the depth of thermal stability. Additionally, Cabeus is one of the coldest PSRs, with diurnal variation in surface temperature between 38.7 and $46.7 \mathrm{~K}$ and subsurface 
temperatures estimated to be $38 \mathrm{~K}$ (12). This means that condensed volatiles in this PSR should remain stable longterm on the surface and at depth. We use regolith volatile abundances estimated by (11) based on the LCROSS plume composition, adjusting $\mathrm{CO}$ and $\mathrm{H}_{2}$ based on our reanalysis of LAMP observations (5). Elemental ratios for volatiles sampled by LCROSS, assuming they were condensed in the regolith, are identified in Fig. 1 as "Condensates" (15).

If the volatiles are stored in clathrates, then the plume composition is a reasonable representation of the volatile abundance in the regolith. This is because all volatiles trapped in clathrates are released together when clathrates become destabilized. The LCROSS elemental ratios for volatiles stored as clathrates are identified in Fig. 1 as "Clathrates" (15). We show in Fig. 2 that clathrates are stable at the temperatures and pressures beneath the surface in the Cabeus PSR.

\section{Volatile Sources}

The potential source or combination of sources for volatiles sampled by LCROSS will depend on the timing for volatile delivery. Cabeus crater is estimated to be 3.5 billion years old (21), providing an upper limit for the age of these volatiles. A lower limit comes from modeling the influence of impact gardening on ice deposits. Based on the abundance of ice detected by LCROSS and the depth probed by the impactor, the volatiles sampled should be from more than $1 \mathrm{Gya}$ (22). Although volcanic outgassing was most active more than $3 \mathrm{Gya}$, some activity continued until $1 \mathrm{Gya}$ (3), sh a volcanic source cannot be ruled out based on deposit age. Throughout its history the Moon has been subject to impacts, with the largest fluxes predating the formation of Cabeus, between 3.5 and 4.6 Gya (23). However, impacts by comets and meteorites have continued since that time at a lower rate. Comets and chondrites in the form of asteroid impactors and micrometeoroids (24) are also a reasonable volatile source. Finally, water molecules can form through surface chemistry initiated by solar wind protons and travel to the PSRs (22).

In Fig. 1 we compare the elemental composition for the LCROSS observations with potential volatile sources (15). Comet composition is based on coma measurements of sublimated ices, and varies significantly. However, refractory material in comet nuclei is likely chondritic in composition, so comet impacts would provide a combination of material with what we designate as "cometary," as well as "chondritic" composition. We provide the composition for the coma of 67P/Churyumov-Gerasimenko (67P/C-G), using the best effort to date at determining elemental composition with Rosetta observations (20). We also illustrate average or extreme values based on coma observations from several comets (20). The C/S measured in comets ranges between 2.2 and 8.0 when sulfurbearing species have been detected. $\mathrm{N} / \mathrm{C}$ in comets ranges between 0.06 and 0.37 . Note that the nitrogen inventory for these comets do not include $\mathrm{N}_{2}$, which is difficult to measure remotely. In 67P/C-G, $\mathrm{N}_{2}$ contributed 17\% of the total nitrogen inventory in the coma. The volcanic composition is from (3) with N/C from (25).

\section{Source Mixtures}

As Fig. 1 shows, no source is a perfect fit for the LCROSS measurements. Volcanic sources and chondrites provide the right amount of sulfur, but do not provide sufficient hydrogen and nitrogen. Volcanic sources are also deficient in oxygen. Comets provide sufficient hydrogen, carbon, and nitrogen, but are depleted in sulfur - even when considering the most extreme value. Solar wind only contributes hydrogen and oxygen (see supplementary materials). We developed a model to determine if a mixture of sources can match the LCROSS observations, and found that no combination was able to match all four elemental ratios within the uncertainties of the LCROSS measurements - even when taking into account the uncertainties for the sources (see supplemental materials). The 
main limitation is fitting both the $\mathrm{C} / \mathrm{S}$ and the N/C ratios observed by LCROSS. The two sources with sufficient sulfur to match $\mathrm{C} / \mathrm{S}$, volcanoes and chondrites, are too depleted in nitrogen and hydrogen for any cometary contribution to provide agreement with $\mathrm{N} / \mathrm{C}$ and still match $\mathrm{C} / \mathrm{S}$. This is the case even using the maximum N/C and the minimum C/S for comets. The best fit is provided by $100 \%$ comets, which agrees with all ratios except for $\mathrm{C} / \mathrm{S}$.

To improve our constraints on the source, or mixture of sources, we consider processes that could fractionate elemental ratios between delivery of the source volatiles to the lunar surface and observation in the LCROSS plume, including volcanic atmospheric processes, impact processes, clathrate formation, and cycles of sublimation and recondensation. Because these processes are complex and difficult to accurately quantify, we determine whether the LCROSS observations represent upper or lower limits for the elemental ratios and summarize the results in Table 1.

\subsection{Volcanic Atmosphere Fractionation}

Volcanic sulfur is thought to be released as $S_{2}$, which could rapidly be lost to the surface as solid elemental sulfur or aerosols before reaching a cold trap (26). This would result in a higher C/S ratio in the PSR compared to the source, so the observed $\mathrm{C} / \mathrm{S}$ is an upper limit for volcanic $\mathrm{C} / \mathrm{S}$. This creates a challenge for explaining the LCROSS $\mathrm{C} / \mathrm{S}$ as volcanic in origin, because volcanic $\mathrm{C} / \mathrm{S}$ would need to be much lower than $\mathrm{C} / \mathrm{S}$ in the LCROSS plume to provide sufficient sulfur to explain the observations.

The relative abundances of elements in volcanic gas can also be changed by the escape of molecules from the top of the atmosphere. Unfortunately, loss rates depend on a wide range of complex parameters that are not well constrained (27), making it difficult to quantify how much elemental ratios can fractionate as a result of escape. However, we can estimate upper and lower limits for LCROSS measurements compared to the sources based on the relative masses of the dominant species for each element. Escape from a volcanic atmosphere would be dominated by $\mathrm{H}$ and $\mathrm{H}_{2}(26,27)$ that either originated in the volcanic gas as $\mathrm{H}_{2}$, or was produced by dissociation of water molecules. This would increase the $\mathrm{C} / \mathrm{H}$ of the volatiles in the PSR, making the observations an upper limit for the source ratio. Atomic oxygen and $\mathrm{OH}$ produced by water dissociation could also be lost, making $\mathrm{O} / \mathrm{C}$ in the PSR a lower limit compared to the source. Any nitrogen present would be in the form of either $\mathrm{N}_{2}$ or $\mathrm{NH}_{3}$, which are either the same mass as or lighter than volcanic carbon-bearing molecules $\mathrm{CO}$ and $\mathrm{CO}_{2}$. This means that the $\mathrm{N} / \mathrm{C}$ in the PSR is a lower limit for N/C in a volcanic source when considering atmospheric escape. Because volcanic N/C is drastically lower than the LCROSS observations, escape does not provide a mechanism allowing for volcanic gas to be the source of nitrogen in the Cabeus PSR.

Although escape of hydrogen and oxygen leads to limits that provide worse agreement between a volcanic source and the LCROSS observations, water produced by solar wind surface chemistry would decrease $\mathrm{C} / \mathrm{H}$ and increase $\mathrm{O} / \mathrm{C}$ over time by adding water to the PSR (28), cancelling out escape fractionation. These ratios would allow for a combination of volcanic and solar wind sources. However, measured N/C and C/S ratios disagree with volcanic source composition, even accounting for processes that change elemental ratios in a volcanically-produced atmosphere, conclusively demonstrating that the volatiles sampled by LCROSS are not from a volcanic source.

\subsection{Fractionation of Impact Material}

Next, we consider fractionation of volatiles delivered by impacts of comets, asteroids, and micrometeoroids. The elemental ratios can be fractionated by impact loss and by escape during transport to cold traps. The total percentage of volatiles retained after impact depends on the impact velocity and angle (29). Volatiles lost to space 
escape rapidly as part of the outward flow of the impact plume. Fractionation is similar to hydrodynamic escape, with preferential loss of lighter species. However, light species flow outward rapidly enough to drag heavier species with them (e.g., 30). Additional loss to space could occur by escape during subsequent transport to cold traps over several Earth days (31). Fractionation can be estimated in the same way as with the volcanic atmosphere, assuming that lighter species are removed at a faster rate than heavier species. Hydrogen would primarily be in light molecules like $\mathrm{H}_{1} \mathrm{H}_{2}$, and water making the $\mathrm{C} / \mathrm{H}$ in the PSR an upper limit compared to $\mathrm{C} / \mathrm{H}$ of the source. Loss of oxygen and $\mathrm{OH}$ would make $\mathrm{O} / \mathrm{C}$ in the PSR a lower limit compared to the source. According to simulations of impact chemistry of comets (32) and chondrites (33), nitrogen in an impact plume would primarily be in the form of $\mathrm{N}_{2}$ with some $\mathrm{NH}_{3}$ present, while carbon and sulfur are found in heavier molecules like $\mathrm{CO}, \mathrm{CO}_{2}, \mathrm{H}_{2} \mathrm{~S}, \mathrm{SO}_{2}$ and OCS. As with the volcanically produced atmosphere, N/C in the LCROSS observations is a lower limit compared to the source. We also note that LCROSS and LAMP did not have the ability to detect $\mathrm{N}_{2}$, which is expected to be produced in impact plumes. The N/C in the LCROSS plume may have been higher than observed, arguing further for that the observation is a lower limit compared to the source. The masses for carbon-bearing species are generally lighter than sulfur-bearing species, suggesting that C/S in the LCROSS observations is a lower limit compared to the source. We applied our model again using these constraints (see Table 1) and found that only cometary ices, with some contribution from solar wind-produced water, can explain all four elemental ratios.

\subsection{Clathrate Formation}

During the cooling of an impact plume, clathrates can form with entrapped mixtures different from the coexisting gases. In this case, the entrapped mixture will be enriched in $\mathrm{H}_{2} \mathrm{~S}$ and $\mathrm{SO}_{2}$, and depleted in $\mathrm{CO}$ compared to the initial mixture because $\mathrm{H}_{2} \mathrm{~S}$ and $\mathrm{SO}_{2}$ have a higher propensity for trapping compared to $\mathrm{CO}$ at low pressure conditions (16). If insufficient water is available to trap all of the $\mathrm{CO}, \mathrm{H}_{2} \mathrm{~S}$ and $\mathrm{SO}_{2}$ present in the gas, $\mathrm{C} / \mathrm{S}$ in the clathrates is lower than in the source. Ammonia is not trapped in clathrates, but would form ammonia hydrates at temperatures between 80 and $100 \mathrm{~K}$, or condense as pure ammonia frost at temperatures below $80 \mathrm{~K}$. If not all of the $\mathrm{CO}$ is trapped, but all of the $\mathrm{NH}_{3}$ ends up in the PSR, the N/C observed by LCROSS is an upper limit compared to the source. In this case either comets or chondrites could agree with the $\mathrm{C} / \mathrm{S}$ and N/C. However, based on the water to $\mathrm{CO}$ ratio in clathrates the $\mathrm{O} / \mathrm{C}$ ratio for volatiles trapped in clathrates must be lower than 6.75 if not enough water was available for all of the $\mathrm{CO}$ to be trapped (15). The LCROSS O/C disagrees with this limit, so additional water must be supplied by the solar wind. We again modeled a combination of sources assuming that the LCROSS C/S and $\mathrm{O} / \mathrm{C}$ are lower limits based on clathrate formation processes and escape, that $\mathrm{C} / \mathrm{H}$ is an upper limit based on escape, and ignoring $\mathrm{N} / \mathrm{C}$ because of the competing influences of clathrate formation and escape. We found that a combination of cometary and solar wind sources fits these constraints, but that the modeled $\mathrm{O} / \mathrm{C}$ is too high to support clathrate formation even accounting for a solar wind water source. Therefore, it is unlikely that ices that formed as clathrates can explain the LCROSS observations.

\subsection{Sublimation and Recondensation}

Finally, we consider how a cycle of sublimation and recondensation of volatiles could fractionate the elemental ratios. As volatiles are transported to the PSR, they could condense to the surface at night and sublimate during the day. A similar cycle could also take place within a PSR if diurnal temperatures vary enough to cause sublimation of some species depending on their volatility. The temperatures in the Cabeus PSR are very low and not likely to cause diurnal variations, but volatiles in this PSR could have been influenced by these processes before being trapped. Additionally, recondensation could occur within the Cabeus PSR when volatiles are released through impact gardening. This cycle would increase the abundance of water relative to other species observed in the LCROSS 
plume that have lower volatility temperatures (5). It would also increase the abundance of $\mathrm{NH}_{3}, \mathrm{H}_{2} \mathrm{~S}$, and $\mathrm{SO}_{2}$ relative to $\mathrm{CO}$ and $\mathrm{N}_{2}$. This means that $\mathrm{C} / \mathrm{S}, \mathrm{N} / \mathrm{C}$, and $\mathrm{C} / \mathrm{H}$ in the PSR are lower limits compared to the source, while $\mathrm{O} / \mathrm{C}$ is an upper limit. We modeled the source contributions with these constraints and found that a combination of comets, chondrites, and solar wind was possible. To narrow the possibilities further we add four more constraints shown in Table 1. Including these constraints limits the possible combination of source volatiles to $30-45 \%$ cometary and $55-70 \%$ chondrites with no solar wind contributions. Finally, we consider the combination of loss to space and a cycle of sublimation and recondensation. In this case the only reliable constraints are C/S, N/C, N/S and $\mathrm{O} / \mathrm{H}$. These constraints allow for any combination of comets and chondrites with no water provided by solar wind.

Table 1

Model constraints and results for determining the possible sources for the LCROSS plume based on understanding of fractionation processes.

\begin{tabular}{|c|c|c|c|c|c|c|}
\hline & No fractionation & $\begin{array}{l}\text { Volcanic } \\
\text { atm. } \\
\text { processes }\end{array}$ & $\begin{array}{l}\text { Impact } \\
\text { and } \\
\text { Escape }\end{array}$ & $\begin{array}{l}\text { Clathrate } \\
\text { formation }\end{array}$ & $\begin{array}{l}\text { Sublimation } \\
\text { and } \\
\text { recondensation }\end{array}$ & $\begin{array}{l}\text { Escape, subl. \& } \\
\text { recondensation }\end{array}$ \\
\hline $\mathrm{C} / \mathrm{S}$ & \multirow{4}{*}{$\begin{array}{l}\text { Within errors of } \\
\text { LCROSS obs. \& } \\
\text { sources }\end{array}$} & Upper limit & $\begin{array}{l}\text { Lower } \\
\text { limit }\end{array}$ & Lower limit & Lower limit & Lower limit \\
\hline $\mathrm{N} / \mathrm{C}$ & & Lower limit & $\begin{array}{l}\text { Lower } \\
\text { limit }\end{array}$ & $\begin{array}{l}\text { Un- } \\
\text { constrained }\end{array}$ & Lower limit & Lower limit \\
\hline O/C & & Lower limit & $\begin{array}{l}\text { Lower } \\
\text { limit }\end{array}$ & $<6.75$ & Upper limit & Unconstrained \\
\hline $\mathrm{C} / \mathrm{H}$ & & Upper limit & $\begin{array}{l}\text { Upper } \\
\text { limit }\end{array}$ & $>0.07$ & Lower limit & Unconstrained \\
\hline N/S & \multirow[t]{4}{*}{$\mathrm{n} / \mathrm{a}$} & \multirow[t]{4}{*}{$\mathrm{n} / \mathrm{a}$} & $\begin{array}{l}\text { Lower } \\
\text { limit }\end{array}$ & \multirow[t]{4}{*}{$\mathrm{n} / \mathrm{a}$} & Lower limit & Lower limit \\
\hline $\mathrm{S} / 0$ & & & $\begin{array}{l}\text { Upper } \\
\text { limit }\end{array}$ & & Lower limit & Unconstrained \\
\hline $\mathrm{S} / \mathrm{H}$ & & & $\begin{array}{l}\text { Upper } \\
\text { limit }\end{array}$ & & Lower limit & Unconstrained \\
\hline $\mathrm{O} / \mathrm{H}$ & & & $\begin{array}{l}\text { Upper } \\
\text { limit }\end{array}$ & & Lower limit & $\begin{array}{l}\text { Constrained by } \\
\text { solar wind } \\
\text { input }\end{array}$ \\
\hline Results & No good fit & No good fit & $\begin{array}{l}\text { Comets \& } \\
\text { Solar } \\
\text { Wind }\end{array}$ & No good fit & $\begin{array}{l}30-45 \% \\
\text { Comets } \\
55-70 \% \\
\text { Chondrites }\end{array}$ & $\begin{array}{l}\text { Comets \& } \\
\text { Chondrites }\end{array}$ \\
\hline
\end{tabular}

\section{Summary And Conclusions}

Because no combination of known sources is able to match the large abundances of both sulfur and nitrogen compared to carbon measured by LCROSS we had to consider fractionation of the elements between delivery of volatiles to the surface of the Moon and trapping in the PSRs. The large nitrogen abundance allows us to rule out a volcanic atmosphere as a source for any of the volatiles even accounting for fractionating process. The fractionation of the elemental ratios by loss of volatiles to space and a cycle of sublimation and recondensation 
allows for a combination of cometary and chondritic material for the volatiles observed by LCROSS. Recognizing that the refractory material in comets is likely chondritic in composition, comets alone are a reasonable source and are likely the primary source of these volatiles.

Measuring the elemental composition and the isotope ratios of the five elements evaluated in this study as a function of depth within the Cabeus PSR would provide constraints on the relative contribution of the solar wind to the water sampled, as well as details about the impactors. Because the isotope ratios of each source differ enough to serve as a tracer of the source, mapping them with depth would allow us to map out the composition of impactors as a function of time. Additionally, noble gas abundances and their isotope ratios are extremely valuable for tracing the sources of volatiles delivered to the Moon (e.g., 34). As humans prepare to return to the Moon (35), we have an unprecedented opportunity to make such measurements in Cabeus and other PSRs. It is essential that future lunar missions have a plan to characterize the elemental and isotopic composition of lunar volatiles as a function of depth as they are accessed prior to converting volatiles to resources needed for human exploration.

\section{Supplemental Material}

\subsection{Reanalysis of LAMP Observations}

The LCROSS plume was observed 30-60 seconds after impact by the Lunar Reconnaissance Orbiter (LRO) Lyman Alpha Mapping Project (LAMP), which detected $\mathrm{H}_{2}$ and $\mathrm{CO}(2,4)$, and for the first 4 minutes after impact by the LCROSS spacecraft which measured the abundance of several additional species relative to water (1). The published abundances from LAMP did not include the observations that were made at the same time or covering the same region of space as the LCROSS measurements. To allow for direct comparison between the two sets of observations, we have reanalyzed the LAMP observations to find $\mathrm{CO}$ and $\mathrm{H}_{2}$ abundances relative to water in the time period relevant to the LCROSS measurements.

The previous analysis of the LAMP data primarily focused on the pulse of vapor that expanded out in a spherical shell from the impact. This shell crossed LAMP's field of view in the period 30-60 s after impact, which was before LRO passed the impact site. LAMP's field of view was still $>50 \mathrm{~km}$ away from the impact site. A second feature was detected as LAMP's field of view passed above the impact site. This feature is more closely associated with the population of vapor detected by the LCROSS shepherding spacecraft. We used the model outlined in (4) to simulate that population of vapor for better comparison with abundances measured by the LCROSS Shepherding spacecraft. With this reanalysis, we find the sublimation rate of CO needed in the model to reproduce LAMP's observed count rate when it encountered the impact site. The model assumes a sublimating source of $\mathrm{CO}$ with starting velocities consistent with a Maxwell-Boltzmann distribution at temperature of $200 \mathrm{~K}$. We determine the model column density as LAMP would detect it looking across the impact site at an altitude spanning from 30-40 km above the surface. To reproduce the LAMP observations, a source rate of $0.1 \mathrm{~kg} / \mathrm{s}$ was required.

The particle dynamics are such that they disperse from the plume center quickly. Thus, we must make an assumption about the time profile of the source rate to relate LAMP measurements at $90 \mathrm{~s}$ after impact to the LCROSS observations continuing until 3 minutes after impact. We assume that the source rate is constant throughout the first 150 seconds, which likely leads to an upper limit. With these assumptions, then the LAMP observations are consistent with $<15 \mathrm{~kg}$ of CO released out of the $\sim 5500 \mathrm{~kg}$ mass of the LCROSS ejecta, or $<5 \%$ relative to water observed by LCROSS.

\subsection{Results}


Table S1 provides the abundances of each species thought to be present in the plume or in the regolith based on the LCROSS observations using (1) for all species except for $\mathrm{CO}$ and $\mathrm{H}_{2}$, which were recalculated as described above in Materials and Methods. We provide two categories of abundances that depend on the type of ice present in the regolith. If we assume that the species measured in the plume were released all at once by the destabilization of clathrates in the regolith, then the plume composition would represent the regolith composition. This category is designated as "clathrates". If we assume that all volatiles are condensed onto the surface individually, they their abundance in the plume is related to their volatility temperature (10). We determine this using the study conducted by (17) but correcting for the abundances of $\mathrm{CO}$ and $\mathrm{H}_{2}$ that were calculated as part of this work. This category is designated as "condensates".

\section{Table S1.}

Molecular abundance of the volatiles observed in the LCROSS impact plume and their predicted abundance in the regolith if the volatiles are stored as condensed material. The abundance in the LCROSS plume was measured by the LCROSS experiment (1) and LRO-LAMP (2). The LAMP observations have been reanalyzed to obtain abundances measured at the same time as the LCROSS abundances (This work). The regolith estimates are based on the analysis of each species volatility temperature conducted by (11) correcting $\mathrm{CO}$ and $\mathrm{H}_{2}$ to agree with LAMP observations. Species in bold are used for the atmospheric escape analysis. 


\begin{tabular}{|c|c|c|c|c|}
\hline & $\begin{array}{l}\text { LCROSS plume (\%) or } \\
\text { "Clathrates" }\end{array}$ & $\begin{array}{l}\text { Regolith }(11)(\%) \text { or } \\
\text { "Condensates" }\end{array}$ & $\begin{array}{l}\text { Volatility temperature } \\
\text { (K) }(10)\end{array}$ & $\begin{array}{l}\text { Mass } \\
(\mathrm{amu})\end{array}$ \\
\hline $\mathrm{H}_{2} \mathrm{O}$ & $100 \pm 27.5(1)$ & $5.60 \pm 1.54$ & 100.8 & 18 \\
\hline $\mathrm{OH}$ & $0.030 \pm 0.001(7)$ & $\mathrm{n} / \mathrm{a}$ & & 17 \\
\hline \multirow[t]{2}{*}{$\mathrm{H}_{2}$} & $6.0 \pm 5.9$ (This work) & $0.050 \pm 0.049$ & & 2 \\
\hline & & (This work) & & \\
\hline $\mathrm{CH}_{4}$ & $0.65 \pm 0.41(7)$ & $0.0030 \pm 0.0019$ & $22.0(36)$ & 16 \\
\hline $\mathrm{C}_{2} \mathrm{H}_{4}$ & $3.12 \pm 2.46(7)$ & $0.020 \pm 0.016$ & 40.0 & 28 \\
\hline \multirow[t]{2}{*}{ co } & $2.00 \pm 1.99$ & $0.0800 \pm 0.0796$ (This work) & 16.8 & 28 \\
\hline & (This work) & & & \\
\hline $\mathrm{CO}_{2}$ & $2.17 \pm 1.38(7)$ & $0.040 \pm 0.025$ & 53.4 & 44 \\
\hline $\mathrm{CH}_{3} \mathrm{OH}$ & $1.55 \pm 7.92(7)$ & $0.10 \pm 0.51$ & 90.0 & 32 \\
\hline S & Not observed & Not observed & $181(11)$ & 32 \\
\hline $\mathrm{H}_{2} \mathrm{~S}$ & $16.75 \pm 2.82(1)$ & $0.200 \pm 0.034$ & 47.8 & 34 \\
\hline $\mathrm{SO}_{2}$ & $3.19 \pm 0.08(7)$ & $0.200 \pm 0.005$ & 70.5 & 64 \\
\hline OCS & Not observed & Not observed & $46.8(36)$ & 60 \\
\hline $\mathrm{NH}_{3}$ & $6.03 \pm 1.26(7)$ & $0.070 \pm 0.034$ & 63 & 17 \\
\hline $\mathbf{N}_{2}$ & Not observed & Not observed & $16.2(36)$ & 28 \\
\hline
\end{tabular}

Table S2 applies the species composition from Table S1 to calculate the elemental abundances for the regolith ices if they are "clathrates" or "condensates". Note that the greatest difference between the elemental abundances for the two types of ice are found in the nitrogen and oxygen relative to carbon. We also include the elemental abundances used for the sources shown in Fig. 1 based on the references indicated in the main article text and in the table references. Uncertainties are propagated from measured values reported in the literature to the elemental ratio uncertainties by standard means for error propagation. These elemental ratios and their uncertainties were used in the model described below for each of the cases outlined in Table 1 of the main text.

\section{Table S2.}

Elemental abundance of the volatiles in the top 1-3 meters of regolith in the Cabeus Crater PSR sampled by the LCROSS impact compared to possible sources for the volatiles. The LCROSS plume abundance assumes that the volatiles are stored as clathrates. The regolith abundance assumes that the volatiles are stored as condensates and that each species is released according to its volatility. 


\begin{tabular}{|c|c|c|c|c|c|c|c|}
\hline & $\begin{array}{l}\text { LCROSS } \\
\text { plume (1) } \\
\text { or } \\
\text { "Clathrates" }\end{array}$ & $\begin{array}{l}\text { Regolith (11) } \\
\text { or } \\
\text { "Condensates" }\end{array}$ & $\begin{array}{l}\text { 67P/C-G } \\
(20)\end{array}$ & $\begin{array}{l}\text { Average } \\
\text { comets } \\
(20)\end{array}$ & $\begin{array}{l}\text { Cl } \\
\text { Chondrites } \\
(38,39)\end{array}$ & $\begin{array}{l}\text { CM } \\
\text { Chondrites } \\
\text { (38) }\end{array}$ & $\begin{array}{l}\text { Volcanoes } \\
(3,25)\end{array}$ \\
\hline $\mathrm{C} / \mathrm{S}$ & $0.63 \pm 0.36$ & $0.66 \pm 0.38$ & $5.35 \pm 0.16$ & $\geq 2.16$ & $0.68 \pm 0.02$ & $0.59 \pm 0.02$ & $0.92 \pm 0.47$ \\
\hline $\mathrm{N} / \mathrm{C}$ & $0.48 \pm 0.24$ & $0.27 \pm 0.14$ & $0.10 \pm 0.05$ & $\leq 0.37$ & $0.085 \pm 0.003$ & $\mathrm{n} / \mathrm{a}$ & $0.006 \pm 0.001$ \\
\hline \multirow[t]{2}{*}{ O/C } & $9.37 \pm 4.36$ & $23.7 \pm 11.0$ & $12.06 \pm 4.06$ & $50.0 \pm 49.7$ & $12.58 \pm 0.38$ & $20.80 \pm$ & $1.02 \pm 0.01$ \\
\hline & & & & & & 0.62 & \\
\hline \multirow[t]{2}{*}{$\mathrm{C} / \mathrm{H}$} & $0.04 \pm 0.03$ & $0.02 \pm 0.01$ & $0.05 \pm 0.02$ & $0.01 \pm$ & $2.35 \pm 0.07$ & $1.69 \pm 0.05$ & $17.04 \pm 5.14$ \\
\hline & & & & 0.0099 & & & \\
\hline
\end{tabular}

\subsection{Model Description}

We modeled the possible mixtures of sources by calculating $\mathrm{C} / \mathrm{S}, \mathrm{N} / \mathrm{C}, \mathrm{O} / \mathrm{C}$, and $\mathrm{C} / \mathrm{H}$ for every combination of percent contribution of volcanic, chondrite, cometary, and solar wind contributions that add up to $100 \%$. All elemental ratios used as input to the model are shown in Table S2 with the exception of solar wind contribution. Each time that we applied the model, we tested 167,002 possible combinations of these sources. In all cases, the solar wind was assumed to contribute only hydrogen and oxygen to the ratios by delivering water to the PSR. This is because the solar wind nitrogen and sulfur are orders of magnitude lower than solar wind hydrogen and any contribution would be negligible compared to other potential volatile sources as seen in the right panel of Fig. 1 . The other sources would deliver all four elements according to their abundances in the source.

The model was applied five times using the constraints outlined in Table 1. We did not apply the model for a volcanic atmosphere because we were only evaluating one source in this case. A volcanic source was allowed in all five cases and no models with volcanic contributions could fit in any of these cases because of the deficiency in nitrogen in volcanic gas. When fitting within the uncertainties, a modeled mixture of sources was determined to be an accurate fit if the simulated elemental ratios and uncertainties overlapped with the observations by LCROSS and the uncertainties in the measurements.

\section{Declarations}

\section{Acknowledgments:}

KEM and DMH acknowledge support by the NASA LRO project through LAMP Subcontract A99129JD. KDR and LOM acknowledge support by the NASA LRO LAMP project. KEM and ALK acknowledge support by NASA RDAP grant 80NSSC19K1306.

\section{References}

1. A. Colaprete et al., Detection of water in the LCROSS ejecta plume. Science 330, 463-468 (2010). doi:10.1126/science.1186986

2. G. R. Gladstone et al., LRO-LAMP observations of the LCROSS impact plume. Science 330, 472-476 (2010). doi:10.1126/science. 1186474 
3. D. H. Needham, D. A. Kring, Lunar volcanism produced a transient atmosphere around the ancient Moon. Earth and Planetary Science Letters 478 175-178 (2017). doi:10.1016/j.epsl.2017.09.002

4. D. M., Hurley et al., Modeling of the vapor release from the LCROSS impact: 2. Observations from LAMP. Journal of Geophysical Research: Planets 117, (2012). doi:10.1029/2011JE003841

5. See supplemental materials Table S1

6. E. A. Fisher et al., Evidence for surface water ice in the lunar polar regions using reflectance measurements from the Lunar Orbiter Laser Altimeter and temperature measurements from the Diviner Lunar Radiometer Experiment. Icarus 292,74-85 (2017). doi:10.1016/j.icarus.2017.03.023

7. S. Li et al., Direct evidence of surface exposed water ice in the lunar polar regions. Proceedings of the National Academy of Sciences 115, 8907-8912 (2018). doi:10.1073/pnas.1802345115

8. G. W. Patterson et al., Bistatic radar observations of the Moon using Mini-RF on LRO and the Arecibo Observatory. Icarus 283 2-19 (2017). doi:10.1016/j.icarus.2016.05.017

9. A. B. Sanin et al., Testing lunar permanently shadowed regions for water ice: LEND results from LRO. Journal of Geophysical Research: Planets 117, (2012). doi:10.1029/2011JE003971

10. Volatility temperature is defined as the temperature at which pure solid evaporates from the surface to vacuum at a rate of $1 \mathrm{~mm} /$ billion years assuming a bulk density of $1 \mathrm{~g} / \mathrm{cm}^{3}$ (36) as calculated by (11) using (37)

11. A. A. Berezhnoy, E. A. Kozlova, M. P. Sinitsyn, A. A. Shangaraev, V. V. Shevchenko, Origin and stability of lunar polar volatiles. Advances in space research 50, 1638-1646 (2012). doi:10.1016/j.asr.2012.03.019

12. D. A. Paige et al., Diviner lunar radiometer observations of cold traps in the Moon's south polar region. Science 330, 479-482 (2010). doi:10.1126/science.1197135

13. R. Woods-Robinson, M. A. Siegler, D. A. Paige. A model for the thermophysical properties of lunar regolith at low temperatures. Journal of Geophysical Research: Planets 124, 1989-2011 (2019). doi: 10.1029/2019JE005955

14. P. O. Hayne, B. T. Greenhagen, M. C. Foote, M. A. Siegler, A. R. Vasavada, D. A. Paige, Diviner lunar radiometer observations of the LCROSS impact. Science, 330, 477-479 (2010). doi:10.1126/science.1197135

15. See supplemental materials Table $S 2$

16. J. I. Lunine, D. J. Stevenson, Thermodynamics of clathrate hydrate at low and high pressures with application to the outer solar system. Astrophysical Journal Supplement Series, 58(3), 493-531 (1985). doi:10.1086/191050

17. F. Hersant, D. Gautier, J. I. Lunine, Enrichment in volatiles in the giant planets of the Solar System. Planetary and Space science, 52(7), 623-641 (2004). doi:10.1016/j.pss.2003.12.011

18. A. H. Mohammadi, D. Richon, Phase equilibria of methane hydrates in the presence of methanol and/or ethylene glycol aqueous solutions. Industrial \& engineering chemistry research, 49(2), 925-928 (2010). doi:10.1021/ie901357m

19. J. K. Mitchell, W. N. Houston, W. D. Carrier III, N. C. Costes, “Apollo soil mechanics experiment S-200.” (NASA Space Sciences Laboratory Series 15, Issue 7, 1974).

20. M. Rubin et al., Elemental and molecular abundances in comet 67P/Churyumov-Gerasimenko. Monthly Notices of the Royal Astronomical Society 489, 594-607 (2019). doi:10.1093/mnras/stz2086

21. A. N. Deutsch, J. W. Head III, G. A. Neumann, Analyzing the ages of south polar craters on the Moon: Implications for the sources and evolution of surface water ice. Icarus 336113455 (2020).

doi:10.1029/2020GL087782

Page $11 / 14$ 
22. D. M. Hurley et al., Two-dimensional distribution of volatiles in the lunar regolith from space weathering simulations. Geophysical Research Letters 39 (2012). doi:10.1029/2012GL051105

23. M. D. Hopkins, S. J. Mojzsis, A protracted timeline for lunar bombardment from mineral chemistry, Ti thermometry and $\mathrm{U}-\mathrm{Pb}$ geochronology of Apollo 14 melt breccia zircons. Contributions to Mineralogy and Petrology 169, 30 (2015). doi:10.1007/s00410-015-1123-x

24. M. Benna, D. M. Hurley, T. J. Stubbs, P. R. Mahaffy, R. C. Elphic, Lunar soil hydration constrained by exospheric water liberated by meteoroid impacts. Nature Geoscience 12, 333-338 (2019). doi:10.1038/s41561-019-0345-3

25. B. Fegley, Thermodynamic models of the chemistry of lunar volcanic gases. Geophysical Research Letters 18, 2073-2076 (1991). doi:10.1029/91GL02624

26. I. Aleinov et al., Modeling a transient secondary paleolunar atmosphere: 3-D simulations and analysis. Geophysical Research Letters 46, 5107-5116 (2019). doi:10.1029/2019GL082494

27. O. J. Tucker, R. M. Killen, R. E. Johnson, P. Saxena. Lifetime of a transient atmosphere produced by lunar volcanism. Icarus 359114304 (2021). doi:10.1016/j.icarus.2021.114304

28. D. Hurley Crider, R. R. Vondrak, Space weathering effects on lunar cold trap deposits. Journal of Geophysical Research: Planets 108 (2003). doi:10.1029/2002JE002030

29. L. Ong, E. I. Asphaug, D. Korycansky, R. F. Coker. Volatile retention from cometary impacts on the Moon. Icarus 207, 578-589 (2010). doi:10.1016/j.icarus.2009.12.012

30. K. E. Mandt, O. Mousis, J. Lunine, D. Gautier. Protosolar ammonia as the unique source of Titan's nitrogen. The Astrophysical Journal Letters 788, L24 (2014).

31. P. Prem, N. A. Artemieva, D. B. Goldstein, P. L. Varghese, L. M. Trafton. Transport of water in a transient impactgenerated lunar atmosphere. Icarus, 255, 148-158 (2015). doi: 10.1016/j.icarus.2014.10.017

32. R. Ishimaru, H. Senshu, S. Sugita, T. Matsui. A hydrocode calculation coupled with reaction kinetics of carbon compounds within an impact vapor plume and its implications for cometary impacts on Galilean satellites. Icarus 210, 411-423 (2010). doi:10.1016/j.icarus.2010.06.016

33. H. Kuwahara, S. Sugita. The molecular composition of impact-generated atmospheres on terrestrial planets during the post-accretion stage. Icarus 257, 290-301 (2015). doi:10.1016/j.icarus.2015.05.007

34. K. E. Mandt et al., Tracing the origins of the ice giants through noble gas isotopic composition. Space Science Reviews 216, 1-37 (2020). doi:10.1007/s11214-020-00723-5

35. Artemis III Science Definition Team Report (2020). https://www.nasa.gov/reports

36. J. A. Zhang, D. A. Paige, Cold-trapped organic compounds at the poles of the Moon and Mercury: Implications for origins. Geophysical Research Letters 36 (2009). doi:10.1029/2009GL038614

37. Fray \& Schmidt.

38. C. M. D. Alexander, Quantitative models for the elemental and isotopic fractionations in chondrites: The carbonaceous chondrites. Geochimica et Cosmochimica Acta, 254, 277-309 (2019). doi:10.1016/j.gca.2019.02.008

39. Lodders, K., Solar system abundances of the elements. In Principles and perspectives in cosmochemistry (pp. 379-417). Springer, Berlin, Heidelberg (2010). doi: 10.1007/978-3-642-10352-0_8

\section{Figures}



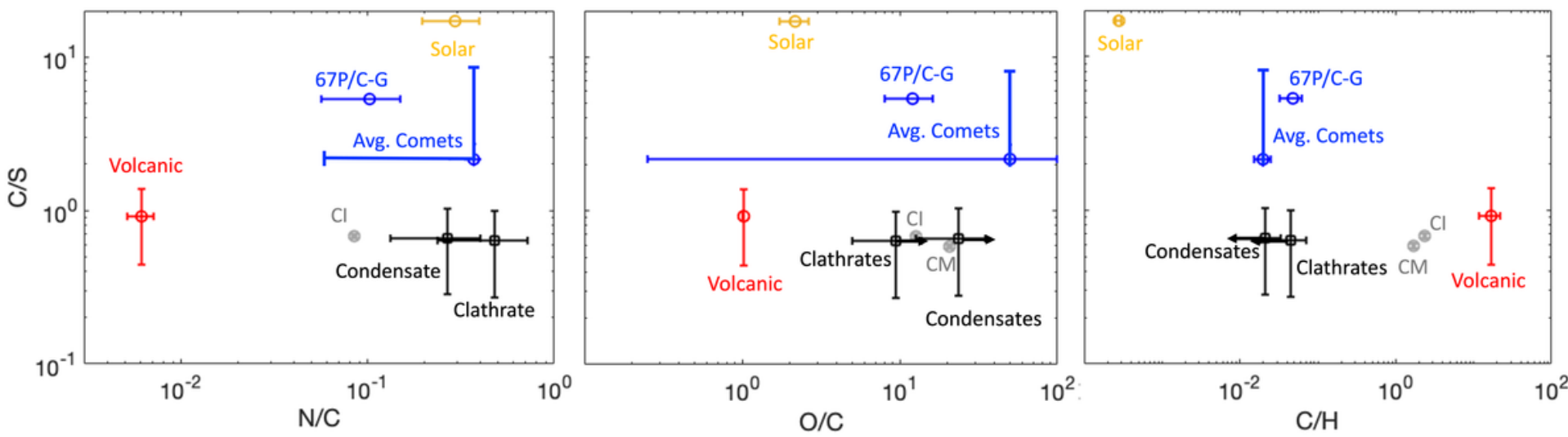

\section{Figure 1}

Elemental composition from LCROSS compared to the elemental composition of possible sources. LCROSS observations (black squares) are based on the assumption that the volatiles are either stored as "Clathrates" or are condensed onto the regolith as "Condensates." Note that no single source exactly matches all of the elemental ratios. Data are provided in (15).

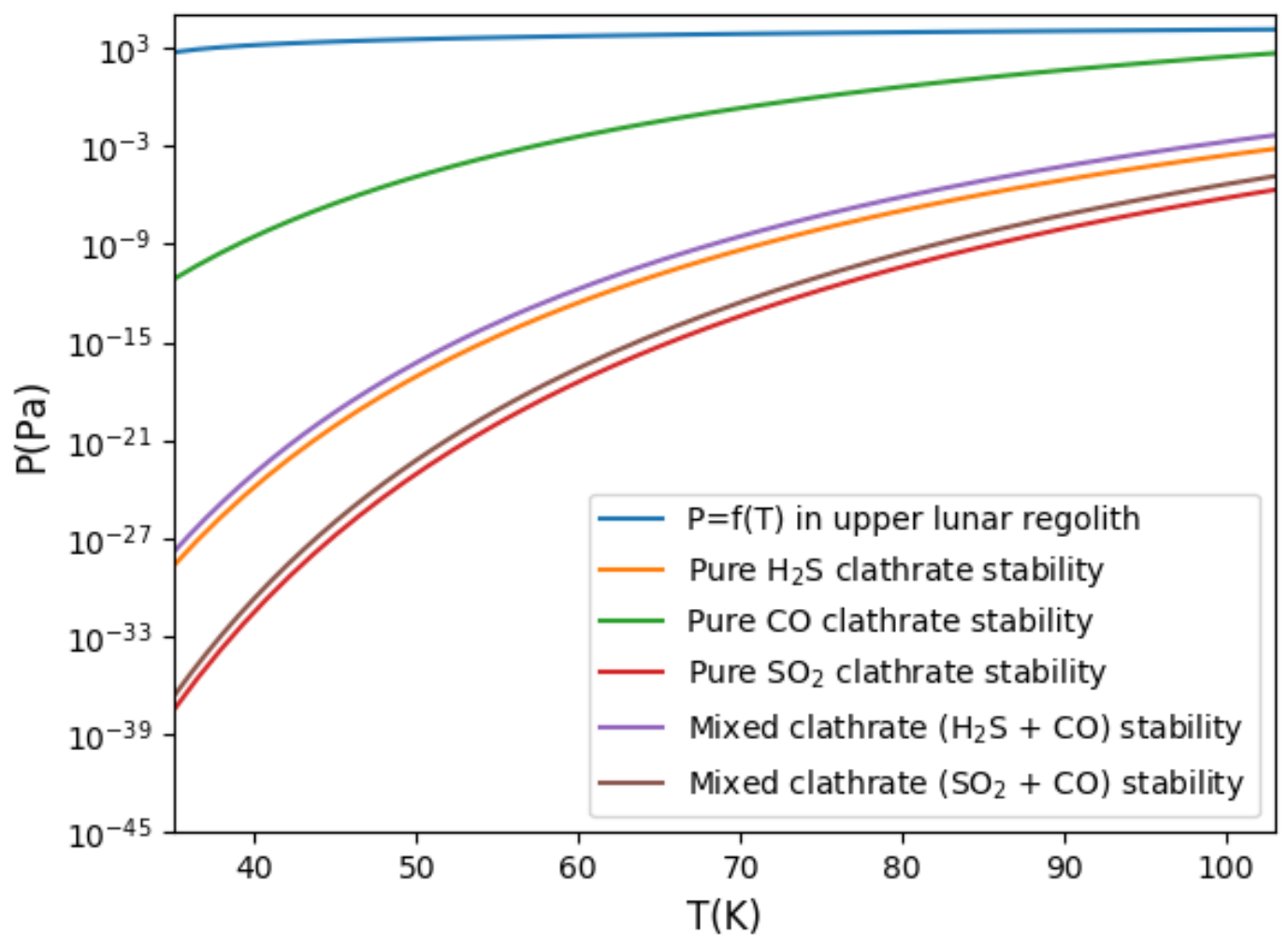

Figure 2

Stability curves for clathrates stored in the PSRs. Comparison of the pressure-temperature profile, or $P=f(T)$, in the upper lunar regolith $(0.2$ to $5 \mathrm{~m})$ to stability curves for clathrates with SO2, H2S, and CO (16-18). The P=f(T) profile is calculated based on a temperature profile extrapolated from (13) and pressure based on a $1.66 \mathrm{~g} / \mathrm{cc}$ lunar 
regolith (19). Mixed clathrate stability curves are based on clathrates formed from gas mixture of $\mathrm{CO}+\mathrm{SO} 2$ or $\mathrm{H} 2 \mathrm{~S}$ (with a cometary $\mathrm{C} / \mathrm{S}$ from 20); such clathrate is dominated by SO2 or H2S. Because the $\mathrm{P}=\mathrm{f}(\mathrm{T})$ for the lunar regolith falls in the area above and to the left of all stability curves, the regolith is within the clathrate stability domain. 